\title{
A histopathological study of gastric mucosal biopsies in infantile hypertrophic pyloric stenosis
}

\author{
GILLIAN BATCUP AND L. SPITZ \\ From the Departments of Pathology and Surgery, The Children's Hospital, Sheffield S10 2TH, UK
}

SUMMARY Gastric biopsies from 35 infants with hypertrophic pyloric stenosis have been assessed for histological features of gastritis. No erosions were found, and there was no excess cellular infiltration of the lamina propria compared with a control group. Fresh haemorrhage into the lamina propria was frequent in both pyloric stenosis and control sections.

The vomitus of infants with hypertrophic pyloric stenosis has been found to contain blood in 3 to $36 \%$ of cases (Benson, 1969; Svensgaard, 1935), with more recent estimates in the region of $18 \%$ (Cook and Rickham, 1978; Dodge, 1975). The bleeding has been variously ascribed to 'irritative gastritis' (Cook and Rickham, 1978; Rickham, 1966), gastritis secondary to gastric stasis (Dodge, 1975; Nixon, 1978), or gastric erosion (Benson and Lloyd, 1964). Inflammation of the oesophagus has also been considered but is deemed to be a much less frequent cause of the haematemesis (Cook and Rickham, 1978).

We report the histological appearances of gastric mucosal biopsies in infants with hypertrophic pyloric stenosis, with particular regard to the presence or absence of gastritis.

\section{Material and methods}

Thirty-five infants with hypertrophic pyloric stenosis were examined with the Olympus oesophagofiberscope Model EP, Type PA (maximum external diameter $7.2 \mathrm{~mm}$ ) under general anaesthesia immediately before operative treatment by pyloromyotomy. All the infants had nasogastric tubes passed preoperatively and gentle washouts of the stomach performed with warm normal saline in order to evacuate the stomach of residual milk curds. This group comprised 28 males and seven females aged 39 to 56 weeks from the time of conception, 10 of whom had vomited fresh or altered blood since admission. A biopsy was taken in each case from the greater curvature of the stomach at a point approximately two-thirds of the distance from the gastrooesophageal junction to the pyloric sphincter.

Received for publication 5 December 1978
Paraffin-embedded blocks were sectioned at $5 \mu \mathrm{m}$ and stained with haemalum and eosin, a modification of the Maxwell trichrome method (Whitehead, 1973), and the Perls' technique for haemosiderin.

The control material was obtained at necropsy examination of 31 infants and two stillbirths ( 21 males and 12 females) ranging in post-conceptional age from 26 to 56 weeks. Within the infant group there were 19 cases of unexpected death (cot death), four of perinatal hypoxia, and one each of acute haemorrhage, pneumonia, precipitate delivery, prematurity, tentorial laceration, intestinal malrotation with cerebral hypoxia, galactosaemia, and injury from a road traffic accident. The majority of children dying unexpectedly showed evidence of a respiratory tract infection, one in addition having Werdnig-Hoffmann disease; three cases in this subgroup without infection were found to have pulmonary oedema, minimal fibroelastosis of the heart, and a metabolic defect respectively. The control cases were selected to exclude any clinical history of diarrhoea or vomiting except as a terminal event in order to obtain gastric mucosa as nearly normal as possible. Cases in which there was marked autolysis of the gastric mucosa were also excluded. At necropsy, fullthickness blocks were taken from the greater curvature of the stomach at a site corresponding to that of the gastric biopsies in the test cases, and were processed and stained in a similar manner.

In both test and control sections, 10 high-power fields were examined. In the test sections these highpower fields were consecutive, excluding areas of traumatic artifact. In the control sections, areas were chosen which showed the least autolysis. The numbers of polymorphs, lymphocytes, plasma cells, and eosinophils within the mucosa were counted, using a $1 \mathrm{~mm}$ square eyepiece graticule, and the results were converted to the numbers of cells per 
$\mathrm{mm}^{2}$ of mucosa. Using all the mucosa available, a qualitative assessment of the presence or absence of haemorrhage, necrosis, or aggregates of inflammatory cells was also made in each case. The modified Maxwell's trichrome stain was used to assess the type of gastric mucosa and the Perls' stain to demonstrate haemosiderin deposits.

\section{Results}

TYPE OF MUCOSA

Of the 35 biopsies from cases of infantile hypertrophic pyloric stenosis, 33 were of body type and two consisted of mucosa transitional in type between pyloric and body zones. All 33 control sections contained body type mucosa.

\section{CELLULAR INFILTRATION}

The numbers of lymphocytes, plasma cells, and eosinophils per $\mathrm{mm}^{2}$ mucosa were plotted against the age from conception, and the results are shown in Figs 1, 2, and 3. There was no significant difference between the numbers of these cells in the mucosa from the cases of pyloric stenosis compared with the control cases. In no instance did the number of polymorphs per $\mathrm{mm}^{2}$ exceed 13 ; in 28 of the pyloric stenosis biopsies and in $\mathbf{2 5}$ of the control stomachs no polymorphs could be identified in the fields examined.

Most of the cellular infiltration was diffuse in type, but small aggregates of cells were also present in the lamina propria. Such aggregates were found in nine of the pyloric stenosis cases and in 25 of the control cases, but the greater volume of mucosa available for study in the latter group would account for this discrepancy. The aggregates consisted mainly of lymphocytes and macrophages, with occasional plasma cells and eosinophils, and were found at all levels in the mucosa (Fig. 4). Cellular aggregates could not be identified in eight of the control cases, and these tended to be younger than average, with ages from conception of $43,47,31,40,26,37,35$, and 39 weeks.

\section{HAEMORR H AGE}

Fresh haemorrhage into the lamina propria was recorded in 18 of the pyloric stenosis group and in 12 of the control group.

\section{HAEMOSIDERIN DEPOSITS}

In no instance was there a conspicuous formation of haemosiderin, and only a very occasional isolated positively staining cell was found in the lamina propria. These cells were seen in only one of the pyloric stenosis biopsies and in two of the control stomachs.
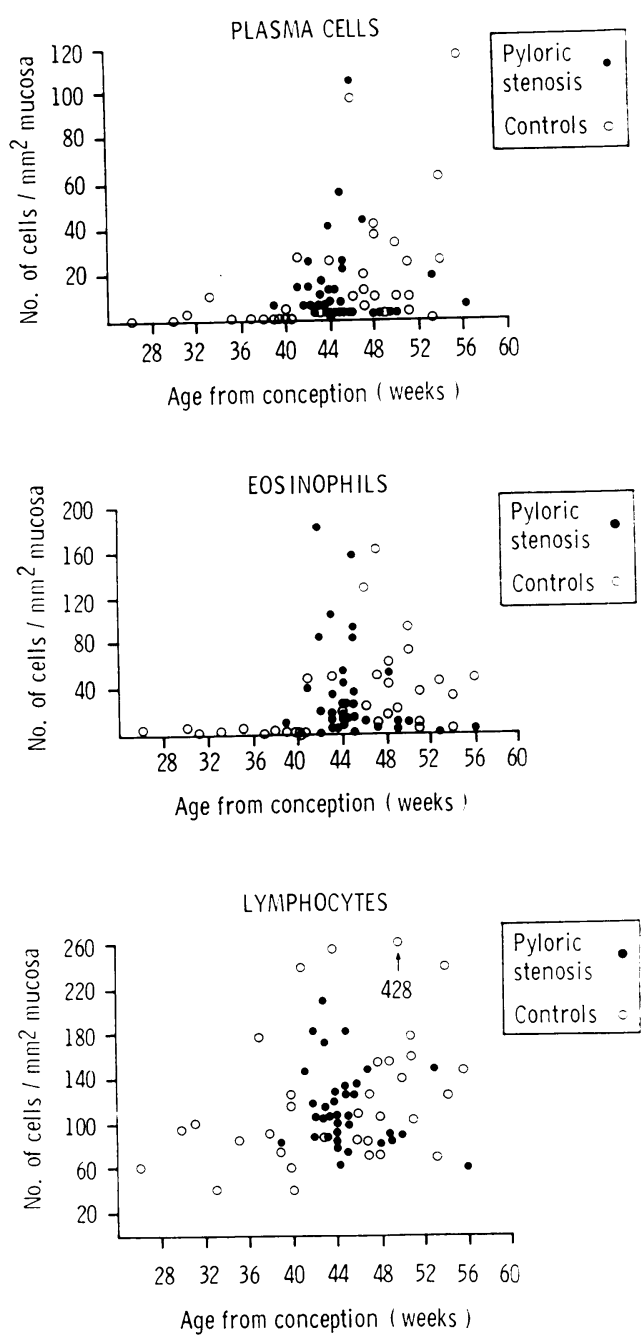

Figs 1, 2, and 3 Numbers of plasma cells, eosinophils, and lymphocytes per square millimetre of gastric mucosa plotted against age from conception.

\section{NECROSIS}

Pyknotic nuclei were occasionally found in epithelial cells in the pyloric stenosis group, where cellular aggregates were situated close to glands, but there was no mucosal ulceration. Acute mucosal erosions with an associated localised polymorph infiltration were present in two of the control sections. One of these was from a premature infant surviving one week with intestinal malrotation and cerebral hypoxia, whose stomach had been aspirated by an indwelling nasogastric tube. The other was from a term infant who died three days after a precipitate delivery. 


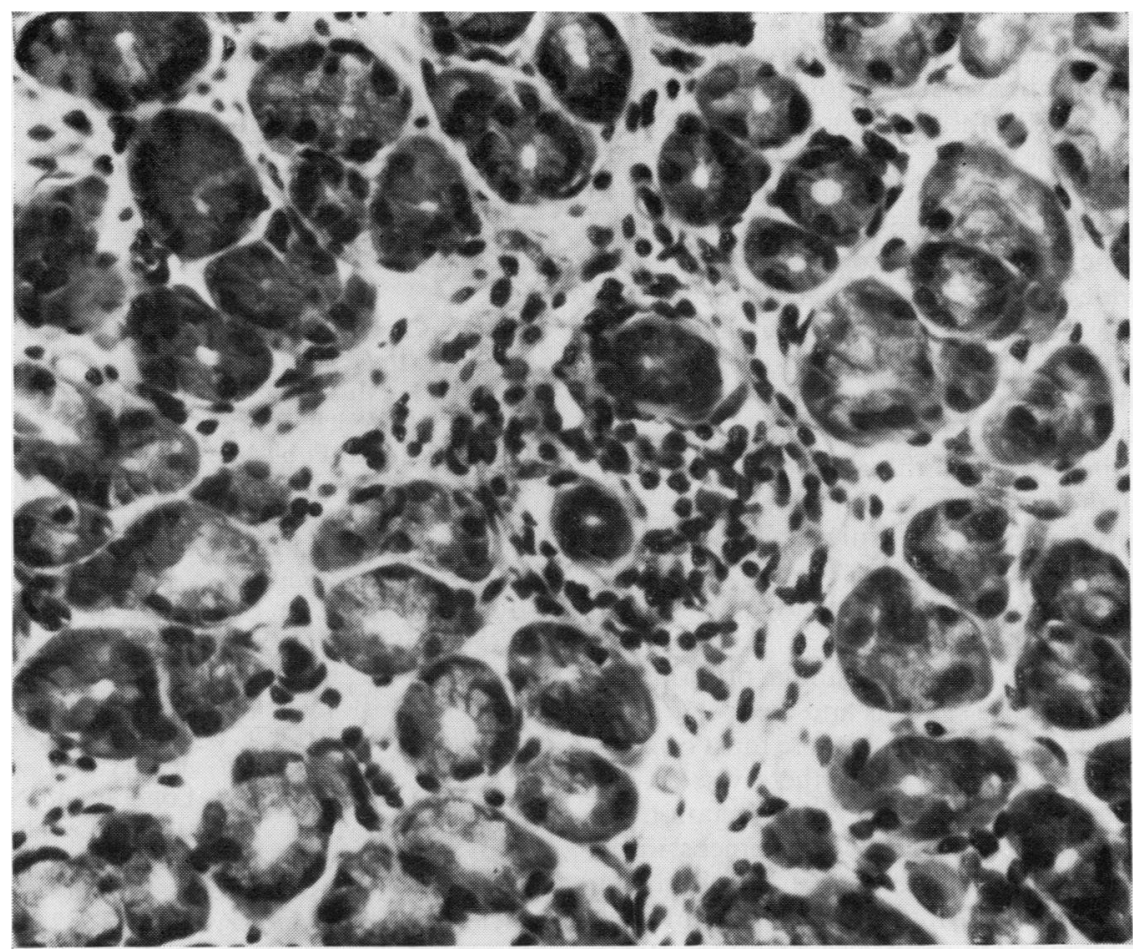

Fig. 4 An aggregate of lymphocytes and histiocytes occupies the centre of the field. Elsewhere in the lamina propria there is a scanty diffuse lymphocytic infiltration (Haemalum and eosin $\times$ 475).

\section{Discussion}

It is generally accepted that the lamina propria of the body zone in the normal adult stomach contains small numbers of scattered plasma cells, lymphocytes, eosinophils, histiocytes, and mast cells (Morson and Dawson, 1972; Whitehead, 1973). Palmer (1954), in his extensive review of gastritis, found additional poorly defined aggregates of round cells in the lamina propria in a group of adult patients who were considered free of gastric disease on clinical grounds. Few studies of the appearances of the gastric mucosa in infants and children have been reported. Faber (1935) found only minimal numbers of mucosal plasma cells and lymphocytes in this age group.

The results of this study revealed no significant difference in the cellular content of the lamina propria between the test and control cases, suggesting that, from the point of view of cellular infiltration, the mucosa of children with infantile hypertrophic pyloric stenosis is normal.

This infiltration appears to be part of the general process of mucosal population of the gastrointestinal tract in response to antigenic stimulation. Within the control group, there was evidence of an increase in the numbers of eosinophils and plasma cells after birth, with a less marked, though similar, trend in lymphocytic infiltration. Similarly, the absence of cellular aggregates tended to be apparent at a younger age within this group. Antigenic stimulation could be provided by formula as opposed to breast feeding. Among the children with infantile hypertrophic pyloric stenosis, seven had been totally breast fed, 23 were partially or completely formula fed, and in five the relevant information was not available. Although the numbers are small, the type of feeding did not appear to influence cellular infiltration significantly.

Acute gastritis from any cause has only rarely become the subject of histological investigation due to the ephemeral nature of the pathology. Where such investigations have been made (Palmer, 1954) the findings have included superficial necrosis, polymorph infiltration and erosions, with an increased number of mucosal lymphocytes, plasma 
cells, and eosinophils after a few days. The biopsies from children with infantile hypertrophic pyloric stenosis examined here were seldom ideally orientated. However, in 33 of the 35 biopsies, the foveolar layer containing the gastric pits was visible, and in 15 of this group the surface epithelium was also included. In none of these biopsies was there evidence of erosions, increased infiltration by inflammatory cells, or widespread necrosis. The occasional pyknotic nuclei close to cellular aggregates may correspond to Palmer's necrobiosis associated with the neck stratum, although others (Whitehead, 1973) have considered his illustrations to represent only traumatic artifacts. Thus these biopsies show no evidence of acute gastritis as defined above. A small biopsy, however, may not be truly representative of the stomach as a whole; the erosions of acute gastritis, for example, are frequently distributed irregularly over the gastric mucosa.

Fresh haemorrhage into the lamina propria was frequent in both pyloric stenosis and control groups. In the controls, the haemorrhages were most obvious at the younger ages, and in many there was an element of severe hypoxia. The interpretation of haemorrhage within the operative specimens introduces a further complication, since it is well known that the procedure of taking a biopsy may in itself induce haemorrhage (Morson and Dawson, 1972). This caveat is, however, most applicable to suction biopsies, and it may be that punch biopsies are less liable to such artifacts. Trauma consequent upon nasogastric intubation and gastric lavage in the patients with pyloric stenosis may also have been responsible for mucosal bleeding. Significant haemorrhage into the lamina propria over several days seems unlikely in view of the paucity of haemosiderin deposition.

True gastric ulcers have rarely been found in association with infantile hypertrophic pyloric stenosis. One such case (Kelsey et al., 1968) presented as a catastrophic haemorrhage in the newborn period, with the subsequent development of classic hypertrophic pyloric stenosis at the age of 3 weeks.

The histological features of chronic gastritis as seen in the adult, such as glandular atrophy and metaplasia, with a marked increase of chronic inflammatory cells in the lamina propria, were absent from both test and control cases.

We thank Mr Gadsdon and his staff for technical assistance, Mrs Gill for typing the manuscript, and Professor J. L. Emery for advice and encouragement.

\section{References}

Benson, C. D. (1969). Infantile hypertrophic pyloric stenosis. In Pediatric Surgery, Vol. 2, 2nd edition, edited by W. T. Mustard, M. M. Ravitch, W. H. Snyder, Jr., K. J. Welch, and C. D. Benson, p. 818. Year Book Medical Publishers, Chicago.

Benson, C. D., and Lloyd, J. R. (1964). Infantile pyloric stenosis. American Journal of Surgery, 107, 429-433.

Cook, R. C. M., and Rickham, P. P. (1978). Gastric outlet obstructions. In Neonatal Surgery, edited by P. P. Rickham, J. Lister, and Irene M. Irving, pp. 335-351. Butterworths, London.

Dodge, J. A. (1975). Infantile hypertrophic pyloric stenosis in Belfast, 1957-1969. Archives of Disease in Childhood, 50, 171-178.

Faber, K. (1935). Gastritis and its Consequences, p. 19. Oxford University Press, London and New York.

Kelsey, D., Stayman, J. W., Jr., McLaughlin, E. D., and Mebane, W. (1968). Massive bleeding in a newborn infant from a gastric ulcer associated with hypertrophic pyloric stenosis. Surgery, 64, 979-982.

Morson, B. C., and Dawson, I. M. P. (1972). Gastrointestinal Pathology, pp. 62 and 80. Blackwell Scientific Publications, Oxford.

Nixon, H. H. (1978). Infantile hypertrophic pyloric stenosis. In Surgical Conditions in Paediatrics, p. 140. Butterworths, London.

Palmer, E. D. (1954). Gastritis: a revaluation. Medicine (Baltimore), 33, 199-290.

Rickham, P. P. (1966). 'Congenital' hypertrophic pyloric stenosis. In Clinical Surgery, vol. 10, edited by C. Rob and R. Smith, pp. 272-283. Butterworths, London.

Svensgaard, E. (1935). The medical treatment of congenital pyloric stenosis. Archives of Disease in Childhood, 10, 443-457.

Whitehead, R. (1973). Mucosal Biopsy of the Gastrointestinal Tract, pp. 2 and 17. W. B. Saunders Company, London.

Requests for reprints to: Dr Gillian Batcup, The Histopathology Department, Children's Hospital, Western Bank, Sheffield S10 2TH, UK. 